Article

\title{
Soft Robotic Gripper with Chambered Fingers for Performing In-Hand Manipulation
}

\author{
Khulan Batsuren and Dongwon Yun * \\ Department of Robotics, DGIST, Daegu 42988, Korea \\ * Correspondence: mech@dgist.ac.kr; Tel.: +82-53-785-6219
}

Received: 5 June 2019; Accepted: 17 July 2019; Published: 24 July 2019

check for updates

\begin{abstract}
In this work, we present a soft robotic gripper for grasping various objects by mimicking in-hand manipulation. The soft robotic gripper consists of three fingers. Each finger contains three air chambers: Two chambers (side chambers) for twisting in two different directions and one chamber (middle chamber) for grasping. The combination of these air chambers makes it possible to grasp an object and rotate it. We fabricated the soft finger using 3D-printed molds. We used the finite element method (FEM) method to design the most effective model, and later these results were compared with results from experiments. The combined experimental results were used to control the range of movement of the whole gripper. The gripper could grasp objects weighing from $4 \mathrm{~g}$ to $300 \mathrm{~g}$ just by inflating the middle chamber, and when air pressure was subsequently applied to one of the side chambers, the gripper could twist the object by $35^{\circ}$.
\end{abstract}

Keywords: pneumatic actuator; soft robotics gripper; FEM

\section{Introduction}

Among many developments in the robotics field, the emergence of soft robotics has dispelled the conventional notion that a robot should be made of rigid materials. Soft robotics have mainly been inspired by muscular hydrostats or hydrostatic skeletons, which are found in the structures of plants and animals such as octopus, snout, snails, and so on [1]. The soft robot is operated pneumatically, hydraulically, or through variable length tendons, by exploiting the soft material's mechanical properties [2,3]. Hard robots have features that pose risks to safety, and design structure flaws including heaviness, high cost, and low thermodynamic efficiency. Soft robots are capable of avoiding these limitations [4-6]. Soft robotics can be used in many fields. An assembled soft robotics gripper made by Soft Robotics Inc. is already being used in the food industry [7]. Depending on the application, a soft robotics gripper can be fabricated by putting several fingers together or fabricated as a single component. Researchers from Harvard have made a starfish-like soft gripper, which is inflated by a single air source. In that study, pneumatic networks called Pneunet chambers were developed, and inflating combinations of the chambers creates complex actuation [8]. All of the elements in this type of robot are actuated simultaneously because they are all supplied by a single air source. Another study presented a soft gripper, which was made by assembling soft actuators that can grasp various objects. This actuator has a bellow, and the study demonstrated a novel approach to actuation based on bellows geometry [9].

The most important factors for a soft actuator that are used in a soft robotic gripper are the air chamber's geometric shape and number of elements as well as the gripper's own structure [10,11]. Researchers have investigated the structure of air chambers and performed a comparison using FEM analysis, finding that the biggest deformation occurred when the top wedged angle was $30^{\circ}$. Also, a comparison of the width of the actuators determined that the thinnest one, at $0.012 \mathrm{~m}$, was easier to actuate during inflation and deflation [12]. However, more air chambers will produce a higher 
degree of freedom. If we apply pressure to all three chambers in a finger simultaneously, the finger will stretch. If one is filled with air, the finger bends, but if two chambers are inflated, it will bend in another direction $[13,14]$.

Considering the material properties of soft actuators, a number of studies have demonstrated that the FEM method is the most appropriate method for analyzing their performance [15,16].

The soft robotic actuator is also more capable of mimicking the manipulation of a human hand compared to a hard robot [17]. Humans have the ability to grasp an object by using the fingers and palm and can translate it from finger to palm or from palm to the fingers, as well as rotate the object. This overall activity is known as "In-Hand Manipulation" [18,19]. In a previous study, researchers developed a soft robotics gripper with three chambers on each soft finger and with the help of flexible sensors, the gripper was able to identify an object's shape, resulting in reliable grasping [20].

Even though some studies have investigated soft robotics grippers with multiple chambers, few studies have examined how changing a longitudinal chamber's location and separation can affect the motion of a soft finger and gripper. In this work, we present a soft robotic gripper, which is able to grasp an object and rotate it (in-hand manipulation) effectively using a combination of three inflatable chambers in a finger. Initially, we compared models with two types of cross-sections and with different chamber locations. As a result, we chose a trapezoid cross-sectioned finger with asymmetrically located chambers. Secondly, we designed a soft gripper, consisting of three soft fingers; each soft finger contains three chambers. The asymmetric location of the chambers allowed two of them to have the same performance while the other one has a different function. By exploiting this feature, we were able to perform two different operations with the soft fingers.

To make a soft finger with many chambers, the fabrication process is typically laborious [21]. But we propose a very simple, time-saving and cost-effective fabrication method using a $3 \mathrm{D}$ printer. Thirdly, given the number of chambers in one finger, we conducted experiments by inflating dual chambers to demonstrate how the finger actuated under various conditions. Later, we compared the experimental results with the FEM data. The pressure data from experiments were used to control the entire gripper. The fabricated gripper was tested by grasping objects that weighed from 0.004 to $0.3 \mathrm{~kg}$.

\section{Analysis of the Soft Actuator}

In this section, we have analyzed the influences of the longitudinal chamber's location and distance apart. Because the deformation characteristics of the actuator depends on the shape of the cross-section, the length of the actuator, and the elastic characteristic of the soft material, the non-linear finite element model is the most effective way to analyze the soft actuator [22]. As can be seen in Figure 1, we considered two different cross-section shapes: Round and trapezoid. The round cross-section is one of the common cross-sectional shape among soft actuators. The studies used round cross-section for their finger, when the finger consisted of a longitudinal single chamber or the chambers are located symmetrically around the central axis $[13,23,24]$. For the finger with a single chamber, the round cross-section is chosen, because the chambers are round themselves. When the chamber is inflated, it extracts the whole outer layer (finger wall) as well as the chamber wall inside layer of the finger. This removes the motion obstacle from the outer layer of finger. We chose the round cross-section as representative of the common shape of soft finger. The trapezoidal cross-section is chosen, assuming the larger contact surface with the object by making a flat surface on each side of the finger. Also, from the studies about the soft finger's cross-sectional shape, it is shown that the finger with a sharp edge performs the highest bending compared to the round cross-sectional shape $[16,25]$. Therefore, we selected the round and trapezoidal cross-sectional shape to investigate the most flexible motion when chambers are located asymmetrically. 


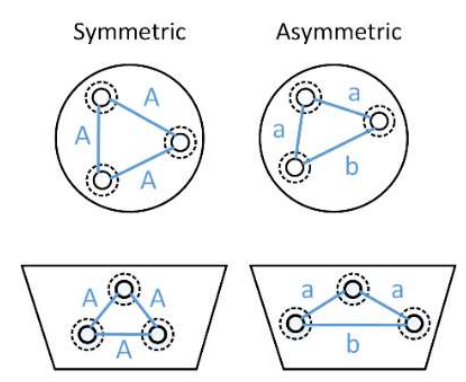

(O) Chambers

Figure 1. Cross-sectional views of the models and distances between chambers.

In each cross-section model, the chambers were located in two different styles: Equally separated from each other (symmetric) and unequally from each other (asymmetric). Each cross-section-shaped finger is investigated under four different distance sizes, including two different distance sizes on both symmetric and asymmetric locations. In total, eight different models are analyzed in this section. All eight models that are compared in FEM analysis have a height of $0.12 \mathrm{~m}$ and the base dimension, wall thickness are changed regarding different distances between the chambers, as shown in Figures 1-3.
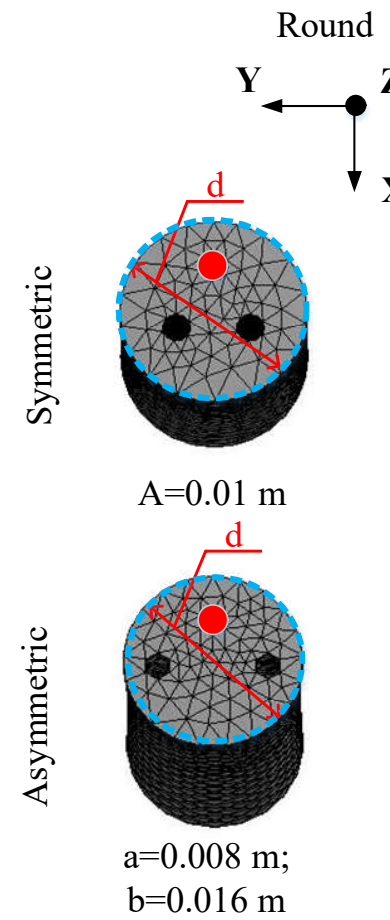

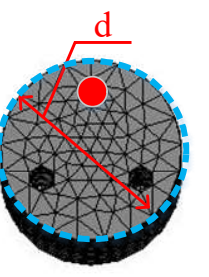

$\mathrm{A}=0.014 \mathrm{~m}$

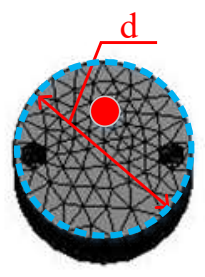

$\mathrm{a}=0.012 \mathrm{~m}$; $\mathrm{b}=0.02 \mathrm{~m}$

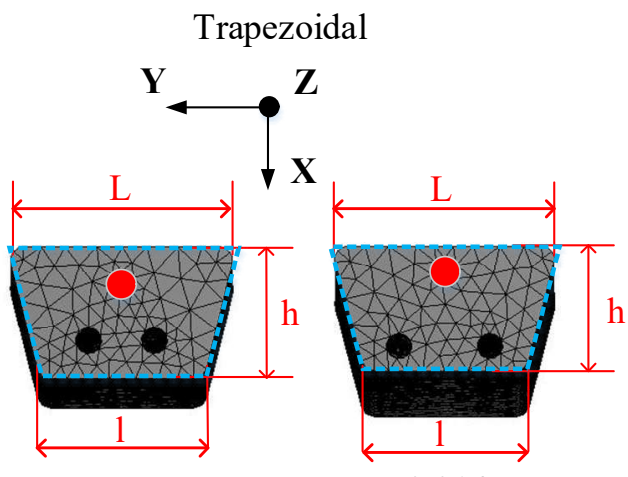

$\mathrm{A}=0.01 \mathrm{~m}$

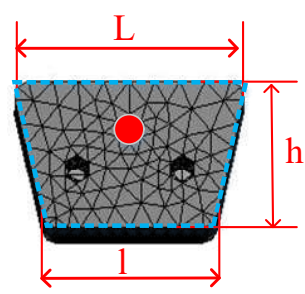

$\mathrm{a}=0.008 \mathrm{~m}$;

$\mathrm{b}=0.016 \mathrm{~m}$
$\mathrm{A}=0.014 \mathrm{~m}$

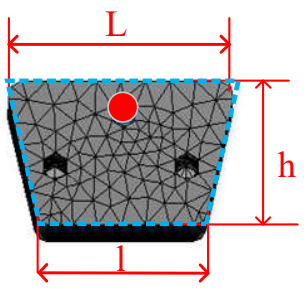

$\mathrm{a}=0.012 \mathrm{~m}$;

$\mathrm{b}=0.02 \mathrm{~m}$

Inflated chamber

Figure 2. Top view of the deformation results in ANSYS when the middle chamber is inflated: Round cross-section with symmetrically located chambers, with chamber distance values of $0.01 \mathrm{~m}$ and $0.014 \mathrm{~m}$ (A value in the figure); round cross-section with asymmetric locations, with chamber distance values of $0.008 \mathrm{~m}, 0.016 \mathrm{~m}, 0.012 \mathrm{~m}$, and $0.02 \mathrm{~m}$ (a,b values in the figure); trapezoidal cross-section with symmetrically located chambers, with chamber distance values of $0.01 \mathrm{~m}$ and $0.014 \mathrm{~m}$ (A value in the figure); trapezoidal cross-section with asymmetrically located chambers, with chamber distance values of $0.008 \mathrm{~m}, 0.016 \mathrm{~m}, 0.012 \mathrm{~m}$, and $0.02 \mathrm{~m}$ (a,b values in the figure). The figure contains the base dimensions of each cross-section. All the round cross-sectioned models have a diameter of $0.026 \mathrm{~m}$ (d) and finger height of $0.12 \mathrm{~m}$, while all the trapezoidal cross-sectioned fingers have a big base length of $0.035 \mathrm{~m}(\mathbf{L})$, a small base length of $0.025 \mathrm{~m}(\mathbf{l})$, and a leg length of $0.02 \mathrm{~m}(\mathbf{h})$. The deformation of each model is shown in the dark part and the dashed line represents the initial position of each finger. 

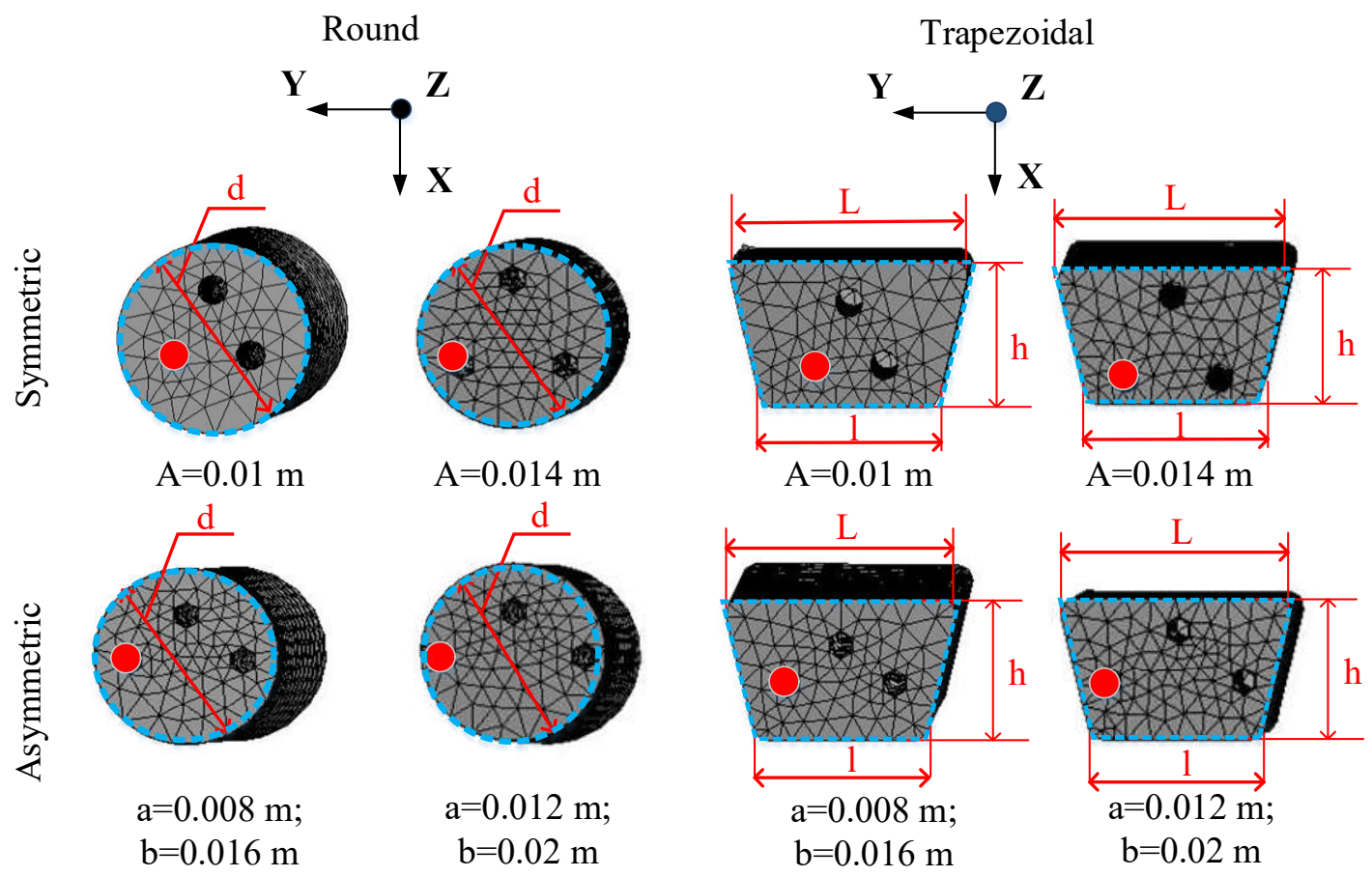

Inflated chamber

Figure 3. Top view showing the deformation results in ANSYS when a side chamber was inflated: Round cross-section with symmetrically located chambers, with chamber distance values of $0.01 \mathrm{~m}$ and $0.014 \mathrm{~m} \mathrm{(A)}$ in the figure; round cross-section with asymmetric locations, with chamber distance values of $0.008 \mathrm{~m}, 0.016 \mathrm{~m}, 0.012 \mathrm{~m}$, and $0.02 \mathrm{~m}(\mathbf{a}, \mathbf{b})$ in the figure; trapezoidal cross-section with symmetrically located chambers, with chamber distance values of $0.01 \mathrm{~m}$ and $0.014 \mathrm{~m}(\mathrm{~A})$ in the figure; trapezoidal cross-section with asymmetrically located chambers, with chamber distance values of $0.008 \mathrm{~m}, 0.016 \mathrm{~m}$, $0.012 \mathrm{~m}$, and $0.02 \mathrm{~m}(\mathbf{a}, \mathbf{b})$ in the figure. The figure contains the base dimensions of each cross-section. All the round cross-sectioned models have a diameter of $0.026 \mathrm{~m}(\mathrm{~d})$ and finger height of $0.12 \mathrm{~m}$, while all the trapezoidal cross-sectioned fingers have a big base length of $0.035 \mathrm{~m}(\mathrm{~L})$, a small base length of $0.025 \mathrm{~m}(\mathbf{l})$, and a leg length of $0.02 \mathrm{~m}$ (h). The deformation of each model is shown in the dark part and the dashed line represents the initial position of each finger.

Every model has three longitudinal chambers. By inflating these three chambers in combination, the finger is able to make motions such as bending, grasping, and twisting, either sequentially or at the same time. But depending on the cross-section shape, the locations of the chambers, and the distance between chambers, the deformation direction and bending angle are all different. In our paper, we focused on the bending angle, which is the angle between the center of a fingertip and its initial position during inflation. In this stage, we optimized the most effective model for making bending and twisting motions. In this paper, a twisting motion is considered to be grasping the object and rotating it by a certain angle by inflating the side chambers. Therefore, we examined the bending angle during each chamber's inflation. We named the chamber that was equidistant from the other two chambers the middle chamber, and the other two chambers were designated side chambers, because those two chambers have the same performance on bending. We assumed that the middle chamber would be used for grasping and that the side chambers would be used for the twisting motion. Thus, to optimize the differences between the symmetric location and asymmetric location of the chambers and to check how it affected the finger's motion, we investigated the middle chamber's inflation and the side chamber's inflation, respectively.

In this part, we focused on analyzing the geometry of the finger using the FEM method. To understand the behavior of the soft actuator, eight different models were analyzed and compared, based on the relationship between pressure and bending angle, by observing the effects of cross-sectional 
shape, the location of chambers, and the distance between chambers. As mentioned previously, we chose two different distance sizes for symmetric and asymmetric styles on each cross-sectional shape: $0.01 \mathrm{~m}$ and $0.014 \mathrm{~m}$ for symmetrically located chambers and the asymmetric chambers involves 0.008 $\mathrm{m}, 0.016 \mathrm{~m}, 0.012 \mathrm{~m}$, and $0.02 \mathrm{~m}$. The space of $0.014 \mathrm{~m}$ and $0.012-0.02 \mathrm{~m}$ was selected due to the minimum wall size of $0.0024 \mathrm{~m}$. If the wall size was smaller than the minimum value, the finger will be easily damaged during high pressure. Also, the difference between each space distance is $0.004 \mathrm{~m}$. The finger's bending motion started to be varied only when we change distance size by $0.004 \mathrm{~m}$. Therefore, we chose the chamber distance sizes with wall size of $0.0024 \mathrm{~m}$ (at minimum) and by variance of $0.004 \mathrm{~m}$.

Figure 2 shows the deformations of all the models, which were formulated in ANSYS Workbench 19.2. In the finite element model, material nonlinearities must be considered in addition to geometry, especially for large deformations of a soft actuator. In the simulation, the material properties were set to be the same as Dragon skin 10 (Smooth-On Inc.), which was later used to make our proposed finger. According to the literature, the incompressible hyperelasticity of Dragon skin 10 is determined to be a third-order Mooney-Rivlin model with coefficients, as shown in Table 1 [26].

Table 1. The incompressible hyperelasticity of the silicone, which is determined to be a third-order Mooney-Rivlin model.

\begin{tabular}{cc}
\hline Name & Coefficient Value (MPa) \\
\hline C10 & 0.04 \\
C01 & -0.033 \\
C11 & $1.2 \times 10^{-3}$ \\
sse & $8.26 \times 10^{-3}$ \\
\hline
\end{tabular}

Firstly, we investigated the middle chamber's inflation. Figure 2 shows the deformation results for all models when the middle chamber was inflated, which resulted in all fingers bending around the $Y$-axis. In other words, when we pressurized the middle chamber, all of the finger models bent in the opposite direction of inflated chamber. As previously mentioned, inflating the middle chamber will be used for grasping. We compared every model's bending angle around the $Y$-axis at given pressures from $25 \mathrm{kPa}$ to $85 \mathrm{kPa}$. As the comparison plot indicates, the symmetrically located chambers led to a larger bending angle at a given pressure. Among all the models, the round cross-section finger with chamber distances of $0.01 \mathrm{~m}$ achieved the highest deformation. It started to bend from $8^{\circ}$ at $25 \mathrm{kPa}$. For the asymmetrically located chambers, the round cross-section finger with chambers distance of $0.012 \mathrm{~m}$ and $0.02 \mathrm{~m}$ displayed the largest deformation. However, when the models with the asymmetric chambers are compared, it can be seen that those models have small differences from each other. These facts imply that if the finger is only used for grasping or bending along one axis, the finger with symmetrically located chambers is the most applicable. However, our final goal is to make a gripper for grasping and twisting, and for this reason, we had to investigate the effect of the side chamber's inflation.

Next, we compared each model's deformation when the side chamber was inflated. As shown in Figure 3, when we applied pressure to the side chamber, the bending direction was different in each model depending on the cross-section shape and the location of chambers. The side chamber's inflation will be used for twisting motion, thus bending around the $X$-axis is the most crucial part in this part. With the round cross-section, the finger bent in the direction away from the inflated chamber. This shows that the round cross-section shape does not produce any obstacle to movement. But in the case of the trapezoidal cross-section, the bending direction differed in each case, based on the chambers' locations. In more detail, when the chambers in the trapezoid finger were located symmetrically around the central axis, most of the bending occurred around the Y-axis. In contrast, in the trapezoidal-shaped finger with asymmetric chambers, rotation occurred around the $X$-axis. The comparison plot of bending angle for every model's side chambers inflation determined that the 
round cross-section with asymmetric chambers, which were separated by $0.012 \mathrm{~m}$ and $0.02 \mathrm{~m}$, and the trapezoidal cross-section with chamber distances of $0.012 \mathrm{~m}$ and $0.02 \mathrm{~m}$, exhibited the highest bending around the $X$-axis, as shown in Figure $4 b$.

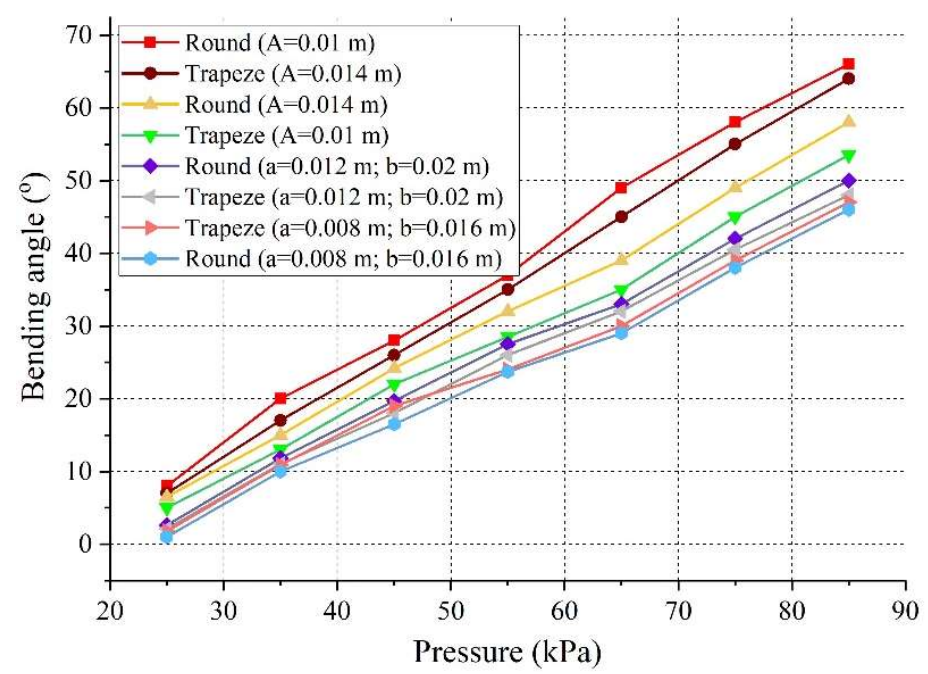

(a)

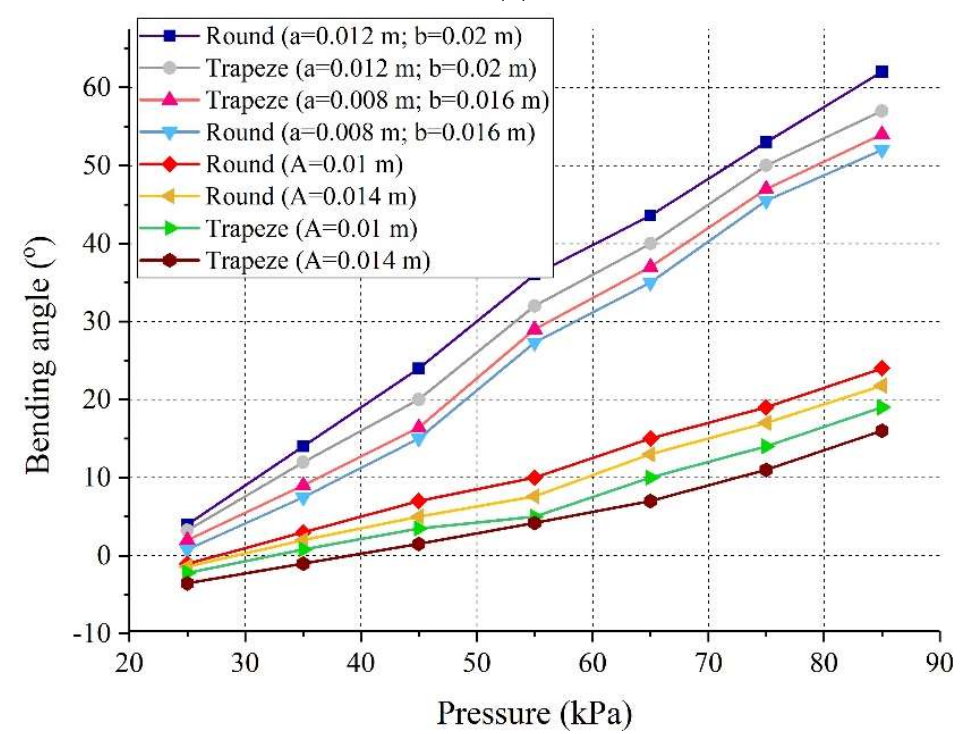

(b)

Figure 4. Comparison of finite element method (FEM) models: (a) Plot showing a comparison of the bending along the $X$-axis for each model, when the middle chamber was inflated. (b) Plot showing a comparison of the bending along the $Y$-axis of each model, when the side chamber was inflated.

Regarding the distance from middle chamber to neutral axis, the deformation of the finger is formed. From the comparison plot of the middle chamber's inflation shown in Figure 4a, we conclude that the distance from the middle chamber to the neutral axis does not directly affect the bending performance. From Table 2, the distance of the round cross-section with symmetric chambers of 0.01 $\mathrm{m}$ showed the largest bending, which is the longest distance from the neutral axis among the round cross-section. Also, if we consider only the symmetric styles, the trapezoid cross-section with the shortest distance to the neutral axis performed the least bending. However, among the asymmetric styles, the round cross-section with distances of $0.006 \mathrm{~m}$ and $0.01 \mathrm{~m}$ from a neutral axis to the middle chamber and side chamber, respectively, showed the highest bending performance. Meanwhile, for the round cross-section with distances of $0.006 \mathrm{~m}$ and $0.008 \mathrm{~m}$ from a neutral axis to the middle chamber and side chamber, respectively, the finger bent with the smallest angle, as can be seen in Figure 4a. 
Table 2. The distance from middle chamber to neutral axis.

\begin{tabular}{cccc}
\hline & & Trapezoid & Round \\
\hline \multirow{2}{*}{ Symmetric } & $\mathrm{A}=0.01 \mathrm{~m}$ & $0.0043 \mathrm{~m}$ & $0.0065 \mathrm{~m}$ \\
& $\mathrm{~A}=0.014 \mathrm{~m}$ & $0.007 \mathrm{~m}$ & $0.0083 \mathrm{~m}$ \\
\hline \multirow{2}{*}{ Asymmetric } & $\mathrm{a}=0.008 \mathrm{~m} ; \mathrm{b}=0.016 \mathrm{~m}$ & $0.0035 \mathrm{~m}$ & $0.006 \mathrm{~m}$ \\
& $\mathrm{a}=0.012 \mathrm{~m} ; \mathrm{b}=0.02 \mathrm{~m}$ & $0.0055 \mathrm{~m}$ & $0.006 \mathrm{~m}$ \\
\hline
\end{tabular}

By combining the distance from each chamber to the neutral axis (Tables 2 and 3) and the side chamber's inflation result from Figure $4 \mathrm{~b}$, we can conclude that the round cross-section with a neutral axis distance of $0.006 \mathrm{~m}$ and $0.01 \mathrm{~m}$ and the trapezoidal cross-section with a neutral axis distance of $0.0055 \mathrm{~m}$ and $0.0095 \mathrm{~m}$ are led by their bending angle result. Whereas, the trapezoidal cross-section with a neutral axis distance of $0.0043 \mathrm{~m}$ and $0.0044 \mathrm{~m}$ and the trapezoidal cross-section with a neutral axis distance of $0.007 \mathrm{~m}$ and $0.006 \mathrm{~m}$ showed low bending performance. The above results demonstrated that the longer distance from chamber to neutral axis can result in higher bending due to thinner wall size, which means the thinner wall leads to the larger inflation of chamber. If the chamber has thin walls, the it is easy to be inflated, thus the finger can bend to a high degree. However, thin walls can also cause the finger to be damaged easily. However, as we mentioned before, we chose the longest distance to neutral axis by considering this side effect, and that the distances of $0.012 \mathrm{~m}$ and $0.02 \mathrm{~m}$ are not enough to be damaged during the inflation.

Table 3. The distance from side chamber to neutral axis.

\begin{tabular}{cccc}
\hline & & Trapezoid & Round \\
\hline \multirow{2}{*}{ Symmetric } & $\mathrm{A}=0.01 \mathrm{~m}$ & $0.0044 \mathrm{~m}$ & $0.0052 \mathrm{~m}$ \\
& $\mathrm{~A}=0.014 \mathrm{~m}$ & $0.006 \mathrm{~m}$ & $0.0071 \mathrm{~m}$ \\
\hline \multirow{2}{*}{ Asymmetric } & $\mathrm{a}=0.008 \mathrm{~m} ; \mathrm{b}=0.016 \mathrm{~m}$ & $0.0078 \mathrm{~m}$ & $0.008 \mathrm{~m}$ \\
& $\mathrm{a}=0.012 \mathrm{~m} ; \mathrm{b}=0.02 \mathrm{~m}$ & $0.0095 \mathrm{~m}$ & $0.01 \mathrm{~m}$ \\
\hline
\end{tabular}

Therefore, considering the contact surface area and performance of the middle chamber's inflation and side chamber's inflation and larger contact surface area, we chose the trapezoidal cross-section with asymmetric chambers, separated by $0.012 \mathrm{~m}$ and $0.02 \mathrm{~m}$. This finger configuration had the optimum performance for grasping and twisting an object, since the finger has to make contact with the object's other surface. Also, the difference in bending performance of the above models when the middle chambers were inflated was not that big. As a result, we concluded that even if we had chosen the second best-performing finger, the difference in deformation would not result in a big difference in the application.

\section{Design of Pneumatic Actuated Gripper and Finger}

\subsection{Finger Design}

A soft robotic finger is operated by applying air pressure to the chambers. The inflation of the chambers guides the motion of the structure. By controlling the inflation of each chamber, we are able to move the soft robotic finger and achieve various motions. When one chamber is filled with air, the finger bends towards the opposite side. Also, if we apply different pressures simultaneously to multiple chambers, the finger makes a twisting motion.

The prototype of the finger was $0.12 \mathrm{~m}$ in length with $0.04 \mathrm{~m}$ diameter chambers, as shown in Figure 5a. As indicated by its cross-sectional view, the finger has a trapezoid shape, with flat surfaces on each side. The central hole of finger enables it to move freely and faster. With this design, the finger can grasp any object with any shape. Unlike other works, the longitudinal chambers are asymmetrically 
arranged around the center. Because of the triangular distribution of chambers, the finger is capable of moving in three directions with less pressure.



(a)

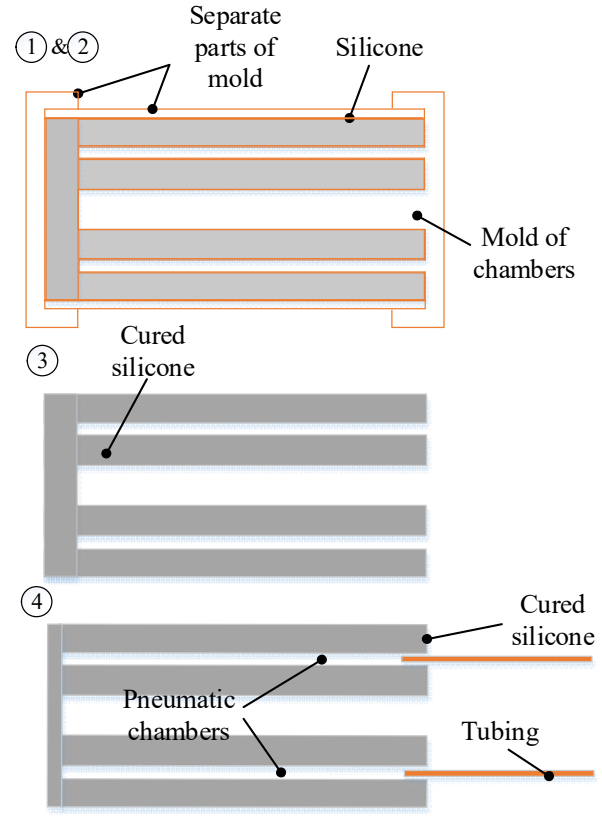

(b)

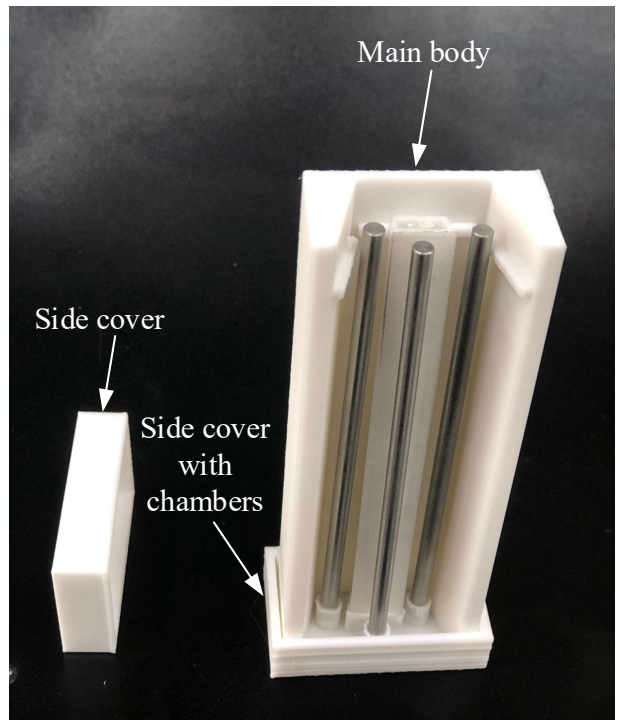

(c)

Figure 5. Soft finger design (a) Computer aided design (CAD) drawing of a proposed finger. (b) Fabrication process for making soft actuator; $(\mathbf{1}, \mathbf{2})$ : Assemble the mold and fill it with liquid silicone; (3): After $5 \mathrm{~h}$ curing, remove actuator from mold; (4): Connect tubing with silicone epoxy. (c) Assembled mold for fabrication. 


\subsection{Fabrication}

Fabrication was performed by the following steps, as shown in Figure $5 b$ :

1. Assemble the separate mold parts: One main body; two side covers including one with chambers, one without chambers, and one cover for protection from dust; and other environmental elements during the cure, as displayed in Figure $5 c$;

2. Fill the assembled mold with silicone and cover the main body part of the mold to protect the finger;

3. Detach all the covers and remove the actuator from the mold after $5 \mathrm{~h}$ curing;

4. Connect tubing to each of the chambers using by silicone epoxy.

Dragon Skin 10 Medium silicone rubber (Smooth On, Inc., Macungie, PA, USA) was used for the silicone material. We chose this silicone for several reasons: The softness and elasticity of the material, the curing time (although the manufacturing process is not difficult, it takes a long time), and its popularity. Also, the plastic mold parts were made with Polylactic acid (PLA) using a 3D printer. Figure 5c shows the four assembled plastic mold parts: The main body, side cover with removable chambers, another side cover, and top cover. These separate molded parts made our fabrication easier and faster. When using plastic for molds, it is difficult to detach silicone. Thus, when curing was finished, we dismantled all the covers and the chambers were removed, lastly. To create a hollow space inside the actuator, we used a similar procedure. A hollow mold was removed with the chambers from the cured actuator. After several attempts, we realized that a horizontal mold would be easier than a vertical one for both assembling and removing components from the actuator (Figure $5 \mathrm{c}$ ). Easy assembly makes the fabrication process more time-saving and cost-effective.

\subsection{Gripper Design}

We considered a soft gripper, comprised of three soft fingers and a hard palm. The three soft fingers were arranged as the thumb, index, and middle fingers, since these are the most essential fingers for in-hand manipulation, as shown as Figure 6c. With the fingers $0.09 \mathrm{~m}$ apart, the gripper will be able to grasp objects with diameters between $0.104 \mathrm{~m}$ and $0.02 \mathrm{~m}$. Figure $6 \mathrm{a}$ shows that the maximum radius of an object that can be grasped is $0.052 \mathrm{~m}$. Figure $6 \mathrm{~b}$ shows the size of the smallest graspable object, approximately $0.01 \mathrm{~m}$. Note that in Figure $6 \mathrm{a}, \mathrm{b}$, the dashed line represents the central axis of the palm. However, the gripper's ability is limited by this value, because the value is defined from the geometric calculation. We used the fabricated gripper with various shaped objects to investigate the limits of its gripping ability. Each finger was mounted on a fitting, hence, the palm was a combination of those fittings and a central part. The separate assembly allows us to modify the number of fingers.

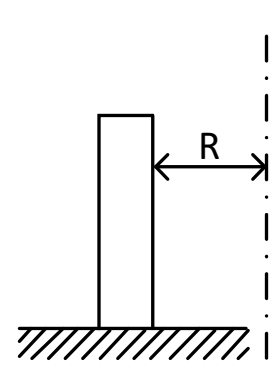

(a)

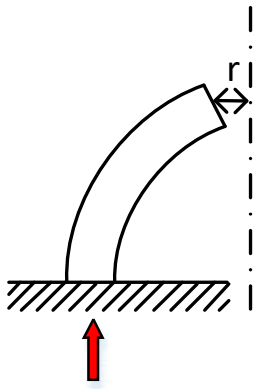

(b)

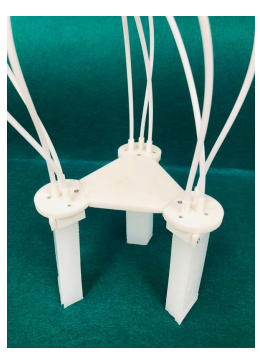

(c)

Figure 6. The gripper design; (a) initial position of a finger: The center of the palm is the dashed line; the maximum grasping size was $\mathrm{R}=0.052 \mathrm{~m}$. (b) Position of a pressurized finger; minimum grasping size $r=0.02 \mathrm{~m}$. (c) The prototype: Fingers are separated by $0.09 \mathrm{~m}$. 


\section{Experiment}

In this section, we present the experimental results of all inflations cases, consisting of both single-chamber inflation and dual-chamber inflation. All experiment results were taken with a V120: Trio, 6DoF optical tracking system with three cameras made by the OptiTrack company. First, we investigated the soft actuator's deformation and measured the bending angle. To measure the bending angle, we set the camera in front of the soft actuator, parallel with the fingertip of the actuator, as can be seen in Figure 8a. We calculated the bending angle with the help of attached markers on the fingertip and the base of the actuator. We did experiments by inflating the middle chamber and side chamber, just as we did in the simulation. We also applied pressure simultaneously to both chambers, as shown in Figure 7. To establish data, we repeated the experiment five times. As illustrated in Figure 7a,b, the actuator is bent by inflating single chambers. Figure 7c shows the dual-chamber inflation cases, including the simultaneous inflation of the middle chamber and side chamber, and sequential inflation, inflating the middle chamber first, and then dual-chamber inflation while the middle chamber was inflated. We include only one figure to illustrate this series, because the results from both cases were the same. This motion is considered twisting.
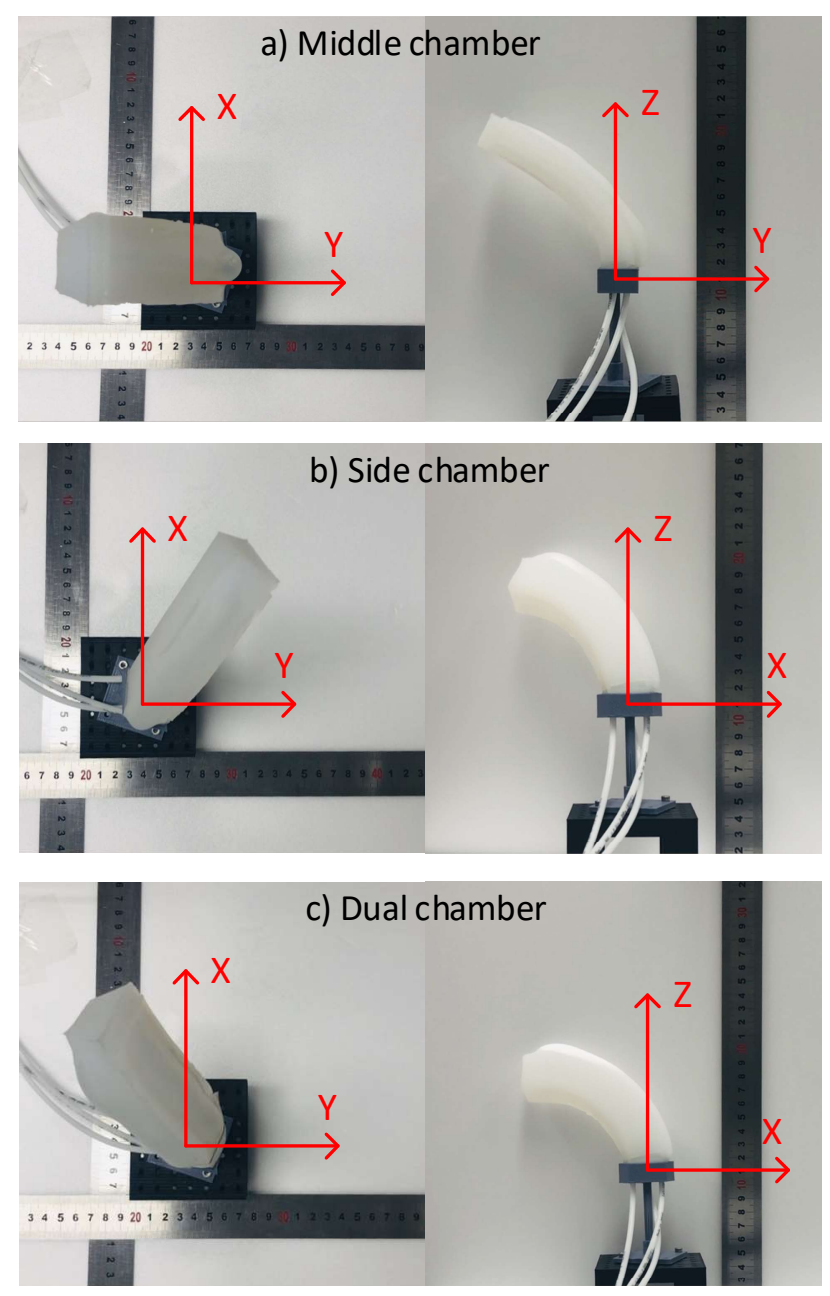

Figure 7. Top and side view of inflations, which include: (a) Middle chamber's inflation, (b) side chamber's inflation, and (c) dual-chamber inflation.

\subsection{Experiment on a Single Actuator}

We conducted experiments by inflating the middle chamber and side chamber just as we did in the simulation. We also applied pressure simultaneously to both chambers, as shown in Figure 7. 
In the experiments, we mostly focused on the bending angle, which is the angle between the center of a fingertip and its initial position during inflation (see Figure 8b). As in the first experiment, we applied pressure ranging from $25 \mathrm{kPa}$ to $95 \mathrm{kPa}$. The deformation of the proposed finger started at $28 \mathrm{kPa}$ and bending angle was resulted in $80^{\circ}$ at $85 \mathrm{kPa}$. We compared the experimental results from the middle chamber's inflation with the results from FEM and noted the difference, which we had assumed would occur, as can be seen in Figure 8c. The difference can be caused by small air losses by components on the pneumatic control board. It can also depend on how we applied pressure to the actuator. In the simulation, all configurations were accurately set, and the software analyzed the motion of finger based on the defined calculation; therefore, the deformation result was stable.

Our next experiment was conducted on the side chamber's inflation. We applied pressures from $25 \mathrm{kPa}$ to $100 \mathrm{kPa}$ and the finger bent fully at $97 \mathrm{kPa}$. Compared to the previous experiment results, the finger bent between two axes. This experiment result was precisely demonstrated in the 3D curve. As shown in Figure 9a, the $\mathrm{O}$ represents the initial position of the fingertip. We combined all of the results from the middle chamber's inflation and side chamber's inflation. From this, we could predict the motion resulting from inflation in both chambers, which means every chamber's inflation will suppress each other's bending motion. To investigate this phenomenon, we conducted the following experiment. We present the last experiment, which was performed by applying the same pressure to both chambers at the same time. Figure $9 \mathrm{~b}$ illustrates the 3D curve after adding the result of the dual chamber's inflation to previous results. We can easily predict the fingertip position using this combination of experimental results. From Figure 7c, we can see that when we applied the same pressure to the middle chamber and side chamber, the finger bent away from the inflated the side chamber. In other words, as shown in Figure $9 \mathrm{~b}$ with respect to applying pressure to a side chamber, the finger bends along the positive side of the $Y$-axis, but when pressure is applied to dual chambers, including the side chamber that was used before, and the middle chamber, the finger bends along the negative side of the $Y$-axis.

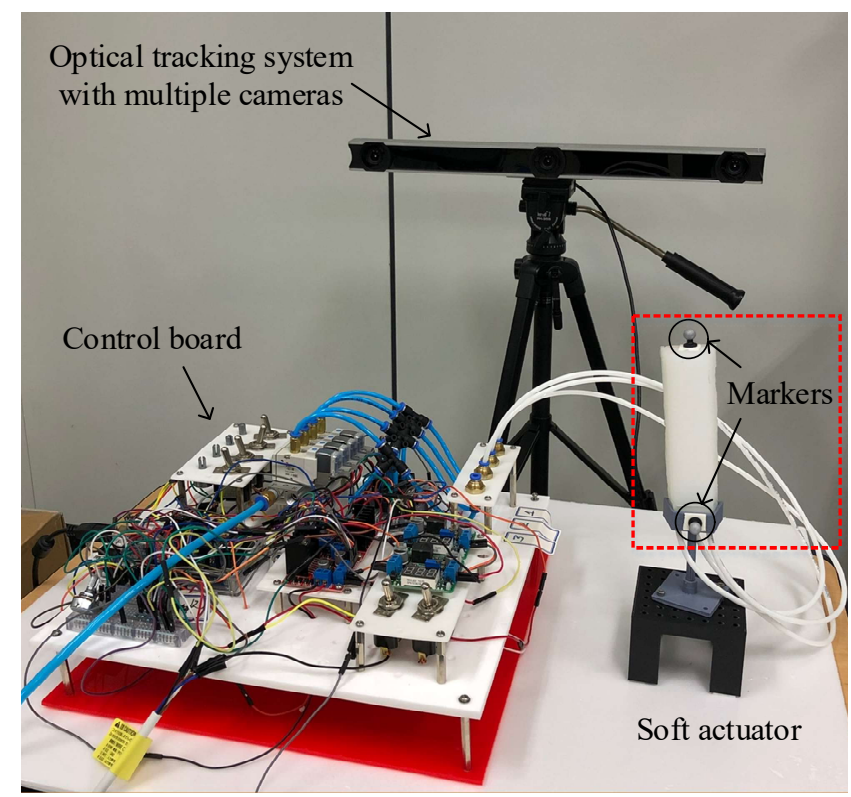

(a)

Figure 8. Cont. 


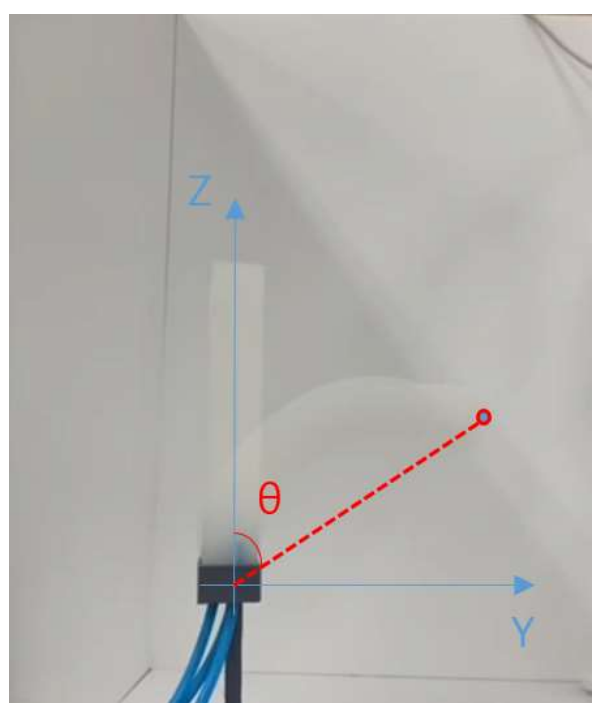

(b)

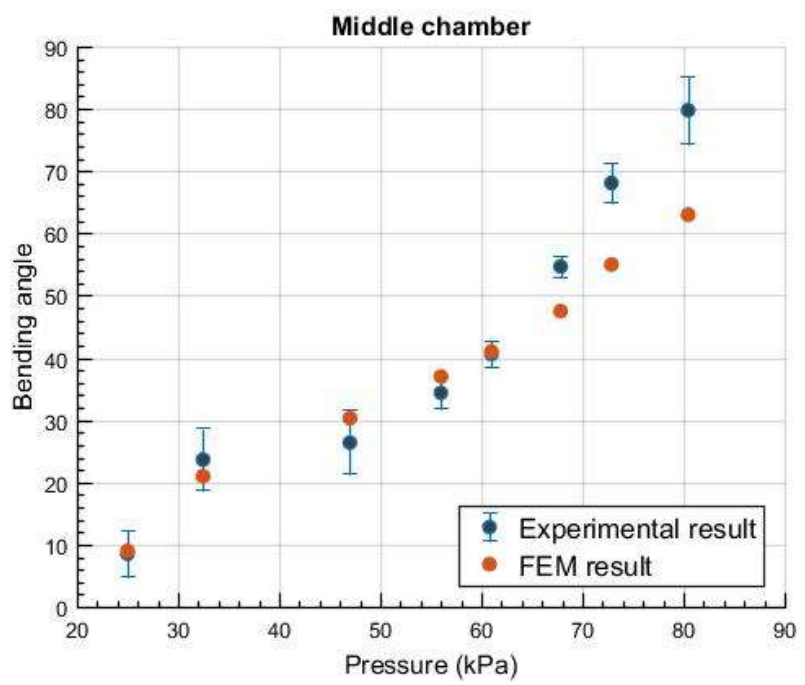

(c)

Figure 8. Experiment on single finger: (a) Experimental setup; (b) bending angle when the middle chamber is inflated; (c) plot showing the relationship between bending angle and pressure with middle chamber's inflation.

We also observed that when we decrease pressure in the air chamber by a certain amount, the finger will bend along the $X$-axis. Following this principle, regarding the ratio of inlet pressure of the middle chamber and side chamber, it resulted in a wide range of workspace as we see in Figure 9. Furthermore, this motion can be used to accomplish the in-hand manipulation. Depending on the object's size, we can use just one side chamber's inflation to rotate the object.

From the 3D curve, we can conclude the following statements:

- Inflating the side chamber can be sufficient to produce a small-angle twisting motion.

- The gripper was found to be capable of grasping bigger objects than expected, as seen in Figure 6, because of the size of the grasp.

- Inflating dual chambers allows smaller objects to be rotated with any angle.

- To grasp and twist sequentially, the dual chamber's inflation is performed after the middle chamber's inflation. 

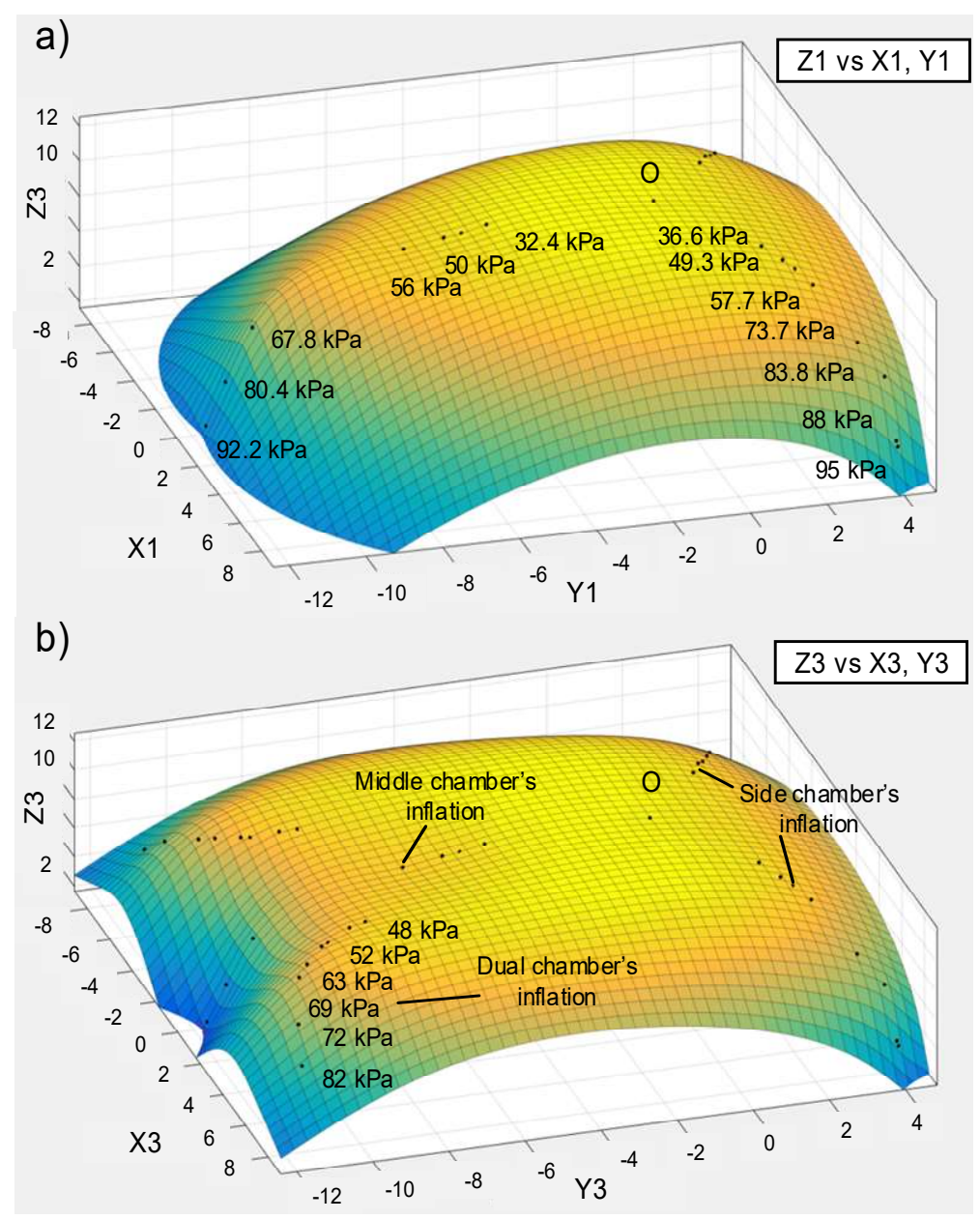

Figure 9. 3D curve demonstrating the position of the fingertip based on results from the experiments: (a) Middle and side chamber's inflation and (b) single- and dual-chamber inflation.

\subsection{Experiment on Gripper}

In this experiment, we tested grasping performance on five objects, which weighed from $0.004 \mathrm{~kg}$ to $0.3 \mathrm{~kg}$. It included objects such as an apple, big and small tape rolls, a square box, and a paper cup, as can be seen in Table 4. We can see from Figure 10a that it grasped all objects successfully. While gripping, the grasping force was increased relative to the weight of an object.

Table 4. The objects used for the experiment. $\mathrm{R}$ is radius, $\mathrm{L}$ is length, and $\mathrm{W}$ is width. The dimensions were measured in the same position as they would be presented during the grasping experiment.

\begin{tabular}{ccc}
\hline Name & Weight $(\mathbf{k g})$ & Object Size $(\mathbf{m})$ \\
\hline Paper cup & 0.004 & $\mathrm{R}=0.035$ \\
Small tape & 0.0405 & $\mathrm{~L}=0.01$ \\
Square box & 0.0475 & $\mathrm{~W}=0.075$ \\
Big tape & 0.1387 & $\mathrm{R}=0.035$ \\
Apple & 0.292 & $\mathrm{R}=0.05$ \\
\hline
\end{tabular}




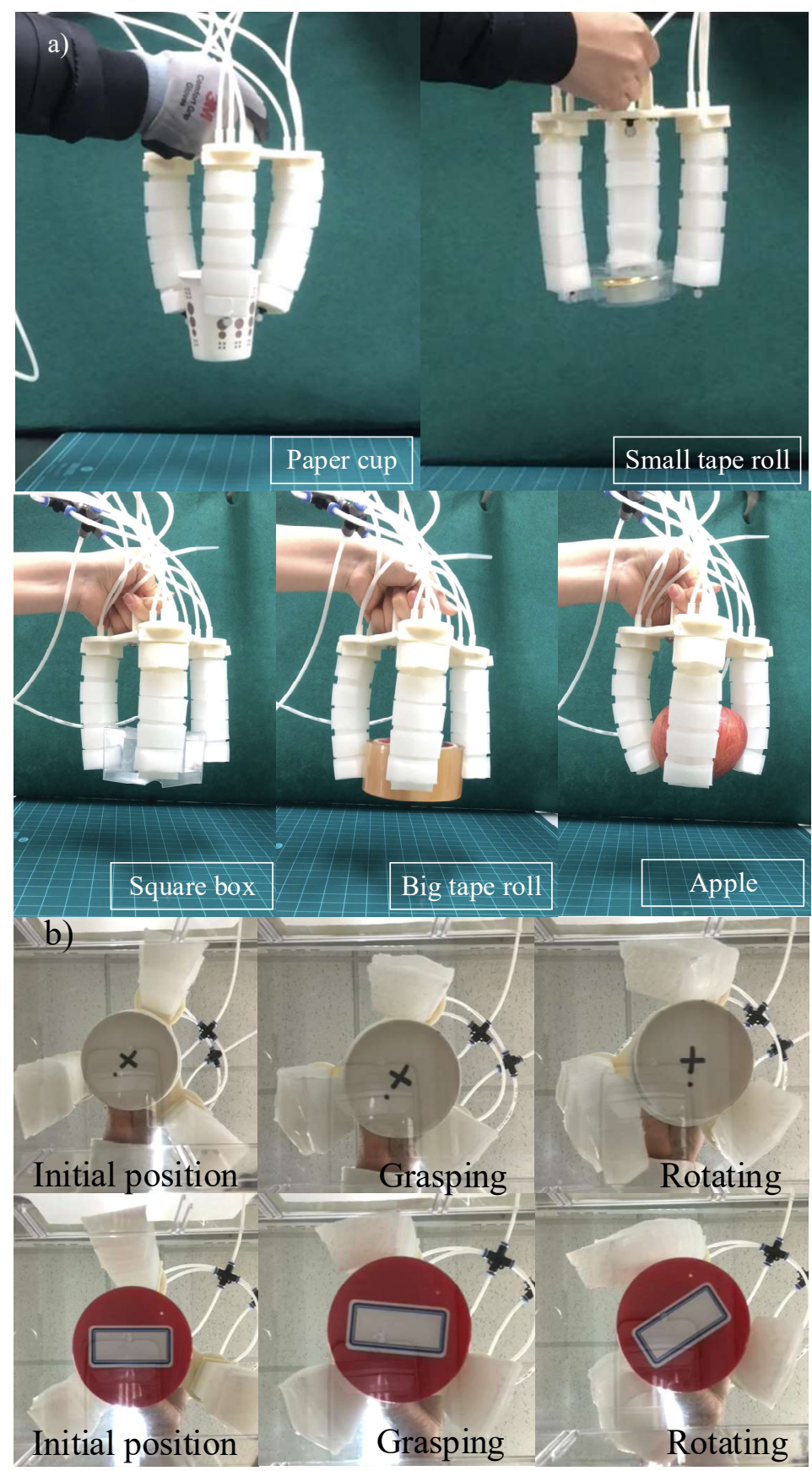

Figure 10. Grasping and twisting experiment: (a) Grasping performance without any object and grasping various objects; (b) bottom view during performance on twisting two different objects: Paper cup $(0.004 \mathrm{~kg})$ and red round box $(0.05 \mathrm{~kg})$.

To complete the experiment on grasping, we used the single finger's experimental data, which were demonstrated in the 3D curve. Each finger's pressure was applied with the same value simultaneously and this worked well on objects like the apple, paper cup, and tape roll, which are round-shaped. This is because the force is applied equally around the object. Also, all of the grasping performances proved our assumption regarding grasping size. The objects were $0.048 \mathrm{~m}$ to $0.010 \mathrm{~m}$ in diameter, which is in the range of the calculated object size.

Lastly, we tested the grasping and twisting performance at the same time. As shown in Figure 10b, to conduct the twisting experiment, we first activated the middle chamber to successfully grasp the target object. After this, we inflated the side chamber to produce twisting while the middle chamber 
was inflated, with the result that the gripper rotated the object. We made a mark on the bottom of each object to see the rotation and measure the twisting angle. From the measurements performed with the $3 \mathrm{D}$ camera, the gripper grasped the objects and rotated the paper cup by $30^{\circ}$ and the round box by $35^{\circ}$.

\section{Conclusions}

In this work, we demonstrated an approach to in-hand manipulation using multiple inflatable air chambers in a soft finger. To predict the soft finger's structure, we prepared two nonlinear finite element models, one with a round cross-section and one with a trapezoidal cross-section. After analysis, we chose trapezoid-shaped finger due to the performance of middle chamber's inflation and side chamber's inflation. We employed a mold for the chosen finger, which reduced many fabrication steps that saved the time and cost. Next, we conducted experiments on a single finger and compared the results with FEM's results. We presented the results as a 3D curve, so we could predict the motion or the position of the fingertip for a given applied pressure. The gripper experiment confirmed that the proposed gripper can grasp an object weighing of $0.3 \mathrm{~kg}$. Also, when we applied pressure to the side chamber after grasping, it could twist the grasped object by $35^{\circ}$ as the largest twisting angle.

We will work on improving the grasping force of our gripper. During the experiments, the friction between the gripper and the object was not high enough to grasp some objects made of plastic or glass. We will expand this performance by adding additional components or modifying the material in the soft finger.

Furthermore, the gripper can be constructed with a manipulator by integrating the microcontroller of gripper with manipulator's controller. The gripper's mechanism is shaped by a few main pieces of equipment, such as valves and pressure sensors, which are connected to the microcontroller. Therefore, the soft gripper can be easily incorporated with manipulator.

Author Contributions: K.B. designed and manufactured the soft gripper. And she conducted the experiment and analyzed data. D.Y. conceived the idea and directed research activities. He also analyzed the analytical and experiment results with K.B.

Funding: This Research was supported by “Development of shape-adaptive electro-adhesive gripper for grasping arbitrary shaped objects" through the Ministry of Trade, Industry and Energy (MOTIE) (10077643).

Conflicts of Interest: The authors declare no conflict of interest.

\section{References}

1. Trivedi, D.; Rahn, C.D.; Kier, W.M.; Walker, I.D. Soft Robotics: Biological Inspiration, State of the Art, and Future Research. Appl. Bionics Biomech. 2008, 5, 99-117. [CrossRef]

2. Rus, D.; Tolley, M.T. Design, fabrication and control of soft robots. Nature 2015, 521, 467-475. [CrossRef] [PubMed]

3. Rich, S.I.; Wood, R.J.; Majidi, C. Untethered soft robotics. Nat. Electron. 2018, 1, 102-112. [CrossRef]

4. Alin, A.-S.; Eiberger, O.; Grebenstein, M.; Haddadin, S.; Ott, C.; Wimbock, T.; Wolf, S.; Hirzinger, G. Soft robotics. IEEE Robot. Autom. Mag. 2008, 15, 3.

5. Kim, S.; Laschi, C.; Trimmer, B. Soft robotics: A bioinspired evolution in robotics. Trends Biotechnol. 2013, 31, 287-294. [CrossRef] [PubMed]

6. Zhou, J.; Chen, S.; Wang, Z. A Soft-Robotic Gripper with Enhanced Object Adaptation and Grasping Reliability. IEEE Robot. Autom. Lett. 2017, 2, 2287-2293. [CrossRef]

7. Bogue, R. Flexible and soft robotic grippers: The key to new markets? Ind. Robot. Int. J. 2016, 43, $258-263$. [CrossRef]

8. Filip, I.; Mazzeo, A.D.; Shepherd, R.F.; Chen, X.; Whitesides, G.M. Soft robotics for chemists. Angew. Chem. 2011, 123, 1930-1935.

9. Drotman, D.; Jadhav, S.; Karimi, M.; Dezonia, P.; Tolley, M.T. 3D printed soft actuators for a legged robot capable of navigating unstructured terrain. In Proceedings of the 2017 IEEE International Conference on Robotics and Automation (ICRA), Singapore, 29 May-3 June 2017; pp. 5532-5538. 
10. Dónal, H.; Abah, C.; Enriquez, M.V.; Herman, M.; Bennett, G.J.; Vela, E.; Walsh, C.J. Strategies for Overcoming Obstacles to the Wide Dissemination of Soft-Robotic Hardware. IEEE Robot. Autom. Mag. 2017, 1070, 2.

11. Peele, B.N.; Wallin, T.J.; Zhao, H.; Shepherd, R.F. 3D printing antagonistic systems of artificial muscle using projection stereolithography. Bioinspir. Biomim. 2015, 10, 55003. [CrossRef]

12. Wang, T.; Gong, Z.; Yang, X.; Guan, S.; Hao, Y.; Ren, Z.; Wang, H.; Wen, L. Modeling and experiments of a soft robotic gripper in amphibious environments. Int. J. Adv. Robot. Syst. 2017, 14. [CrossRef]

13. Suzumori, K.; Iikura, S.; Tanaka, H. Flexible microactuator for miniature robots. In Proceedings of the IEEE Micro Electro Mechanical Systems (MEMS'91), Nara, Japan, 30 December 1990-2 January 1991; pp. 204-209.

14. Drotman, D.; Ishida, M.; Jadhav, S.; Tolley, M.T. Application-Driven Design of Soft, 3-D Printed, Pneumatic Actuators with Bellows. IEEE/ASME Trans. Mechatron. 2018, 24, 78-87.

15. Suzumori, K.; Endo, S.; Kanda, T.; Kato, N.; Suzuki, H. A bending pneumatic rubber actuator realizing soft-bodied manta swimming robot. In Proceedings of the 2007 IEEE International Conference on Robotics and Automation, Roma, Italy, 10-14 April 2007; pp. 4975-4980.

16. Hu, W.; Rahim, M.; Li, W.; Gursel, A. A structural optimization method for a soft pneumatic actuator. Robotics 2018, 7, 24. [CrossRef]

17. Galloway, K.C.; Becker, K.P.; Phillips, B.; Kirby, J.; Licht, S.; Tchernov, D.; Wood, R.J.; Gruber, D.F. Soft Robotic Grippers for Biological Sampling on Deep Reefs. Soft Robot. 2016, 3, 23-33. [CrossRef] [PubMed]

18. Pehoski, C.; Henderson, A.; Tickle-Degnen, L. In-Hand Manipulation in Young Children: Rotation of an Object in the Fingers. Am. J. Occup. Ther. 1997, 51, 544-552. [CrossRef]

19. Sudsang, A.; Ponce, J.; Srinivasa, N. Grasping and in-hand manipulation: Experiments with a reconfigurable gripper. Adv. Robot. 1997, 12, 509-533. [CrossRef]

20. Shih, B.; Drotman, D.; Christianson, C.; Huo, Z.; White, R.; Christensen, H.I.; Tolley, M.T. Custom soft robotic gripper sensor skins for haptic object visualization. In Proceedings of the 2017 IEEE/RSJ International Conference on Intelligent Robots and Systems (IROS), Vancouver, BC, Canada, 24-28 September 2017; pp. 494-501.

21. Martinez, R.V.; Glavan, A.C.; Keplinger, C.; Oyetibo, A.I.; Whitesides, G.M. Soft Actuators and Robots that Are Resistant to Mechanical Damage. Adv. Funct. Mater. 2014, 24, 3003-3010. [CrossRef]

22. Chenevier, J.; González, D.; Aguado, J.V.; Chinesta, F.; Cueto, E. Reduced-order modeling of soft robots. PLoS ONE 2018, 13, e0192052. [CrossRef]

23. Elgeneidy, K.; Lohse, N.; Jackson, M. Bending angle prediction and control of soft pneumatic actuators with embedded flex sensors-A data-driven approach. Mechatronics 2018, 50, 234-247. [CrossRef]

24. Connolly, F.; Polygerinos, P.; Walsh, C.J.; Bertoldi, K. Mechanical Programming of Soft Actuators by Varying Fiber Angle. Soft Robot. 2015, 2, 26-32. [CrossRef]

25. Polygerinos, P.; Wang, Z.; Overvelde, J.T.B.; Galloway, K.C.; Wood, R.J.; Bertoldi, K.; Walsh, C.J. Modeling of Soft Fiber-Reinforced Bending Actuators. IEEE Trans. Robot. 2015, 31, 778-789. [CrossRef]

26. Low, J.H. Customizable Soft Pneumatic Gripper Devices. Ph.D. Thesis, National University of Singapore, Singapore, 2015.

(C) 2019 by the authors. Licensee MDPI, Basel, Switzerland. This article is an open access article distributed under the terms and conditions of the Creative Commons Attribution (CC BY) license (http://creativecommons.org/licenses/by/4.0/). 